\title{
Normality degrees of finite groups
}

\section{MARIUS TĂRNĂUCEANU}

\section{ABSTRACT.}

In this paper we introduce and study the concept of normality degree of a finite group $G$. This quantity measures the probability of a random subgroup of $G$ to be normal. Explicit formulas are obtained for some particular classes of finite groups. Several limits of normality degrees are also computed.

\section{REFERENCES}

[1] Calhoun, W. C., Counting subgroups of some finite groups, Amer. Math. Monthly, 94 (1987), 54-59

[2] Grätzer, G. General lattice theory, Academic Press, New York, 1978

[3] Gustafson, W. H., What is the probability that two group elements commute?, Amer. Math. Monthly, 80 (1973), 1031-1034

[4] Hupert, B., Endliche Gruppen, I, II, Springer Verlag, Berlin, 1967, 1968

[5] Lescot, P., Sur certains groupes finis, Rev. Math. Spéciales, 8 (1987), 276-277

[6] Lescot, P., Degré de commutativité et structure d'un groupe fini (1), Rev. Math. Spéciales, 8 (1988), 276-279

[7] Lescot, P., Degré de commutativité et structure d'un groupe fini (2), Rev. Math. Spéciales 4 (1989), 200-202

[8] Lescot, P., Isoclinism classes and commutativity degrees of finite groups, J. Algebra, 177 (1995), 847-869

[9] Lescot, P., Central extensions and commutativity degree, Comm. Algebra, 29 (2001), 4451-4460

[10] Miller, M. D., On the lattice of normal subgroups of a direct product, Pacific J. Math., 60 (1975), 153-158

[11] Rusin, D. J., What is the probability that two elements of a finite group commute?, Pacific J. Math., 82 (1979), 237-247

[12] Schmidt, R., Subgroup lattices of groups, de Gruyter Expositions in Mathematics 14, de Gruyter, Berlin, 1994

[13] Sherman, G., What is the probability an automorphism fixes a group element?, Amer. Math. Monthly, 82 (1975), 261-264

[14] Suzuki, M., Group theory, I, II, Springer Verlag, Berlin, 1982, 1986

[15] Tărnăuceanu, M., Actions of finite groups on lattices, Seminar Series in Mathematics, Algebra 4, Univ. "Ovidius", Constanţa, 2003

[16] Tărnăuceanu, M., Groups determined by posets of subgroups, Ed. Matrix Rom, Bucureşti, 2006

[17] Tărnăuceanu, M., A new method of proving some classical theorems of abelian groups, Southeast Asian Bull. Math., 31 (2007), 1191-1203

[18] Tărnăuceanu, M., Subgroup commutativity degrees of finite groups, J. Algebra, 321 (2009), 2508-2520

[19] Tărnăuceanu, M., An arithmetic method of counting the subgroups of a finite abelian group, Bull. Math. Soc. Sci. Math. Roumanie (N.S.), 53 (101) (2010), 373-386

[20] Tărnăuceanu, M., Addendum to "Subgroup commutativity degrees of finite groups", J. Algebra, 337 (2011), 363-368

[21] Tărnăuceanu, M., The normal subgroup structure of ZM-groups, Ann. Mat. Pura Appl., 193 (2014), 1085-1088

FACULTY OF MATHEMATICS

"AL. I. CUZA" UNIVERSITY

IAŞI 700506, ROMANIA

E-mail address: tarnauc@uaic.ro

Received: 17.02.2016; In revised form: 13.06.2016; Accepted: 10.07.2016

2010 Mathematics Subject Classification. 20D60, 20P05, 20D30, 20F16, $20 \mathrm{~F} 18$.

Key words and phrases. Normality degree, subgroup lattice, normal subgroup lattice, fixed point. 